\title{
In Situ Preparation of Composite Redox-Active Micelles Bearing Pd Nanoparticles for the Reduction of 4-Nitrophenol
}

\author{
Jiayi You ${ }^{\mathrm{a}}$, Ian Manners ${ }^{\mathrm{b}}$, Hongjing Dou*a \\ a The State Key Laboratory of Metal Matrix Composites, School of Materials Science and Engineering, \\ Shanghai Jiao Tong University, Shanghai 200240, P. R. China. \\ ${ }^{b}$ Department of Chemistry, University of Victoria, Victoria, Bristish Columbia V8P 5C2, Canada. \\ *E-mail:hjdou@sjtu.edu.cn
}




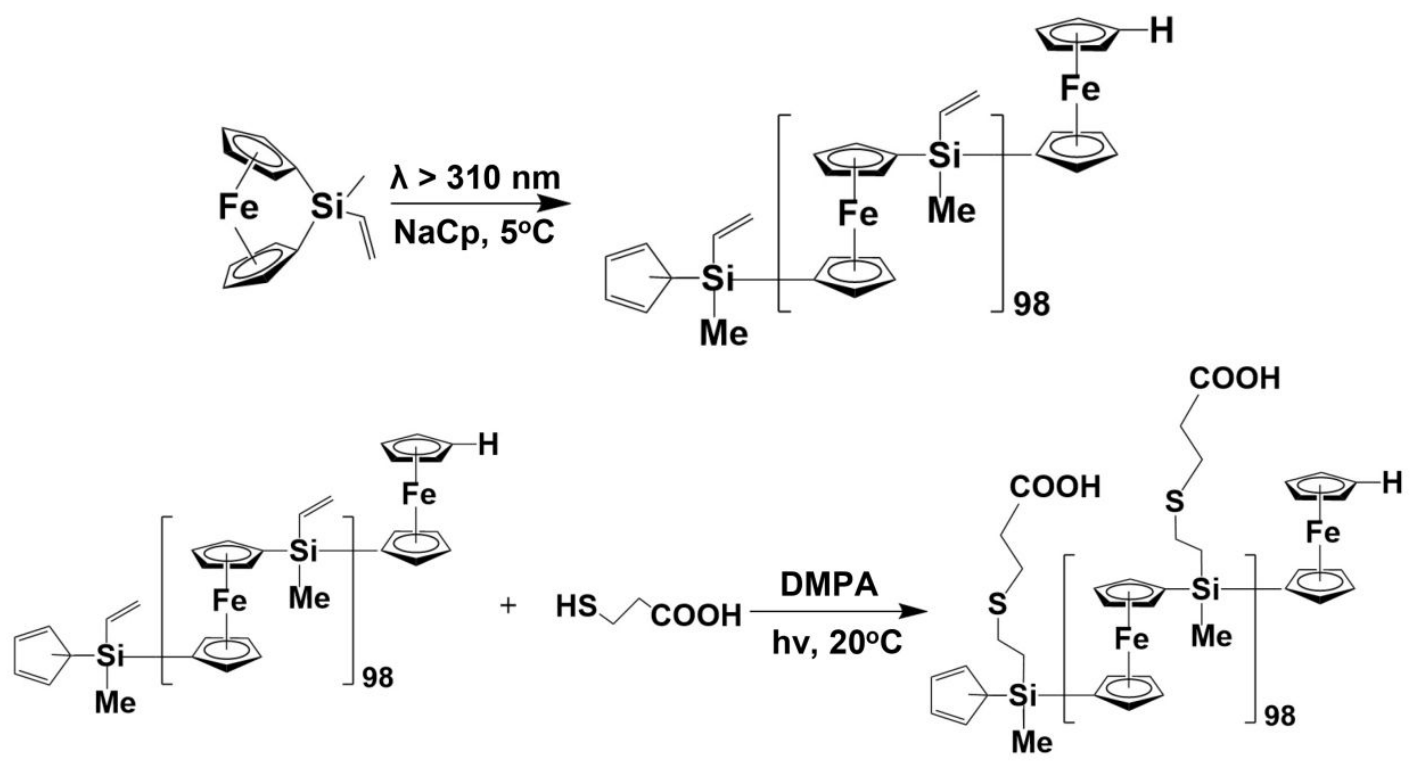

Scheme S1 Synthetic route toward the PFC homopolymer. 

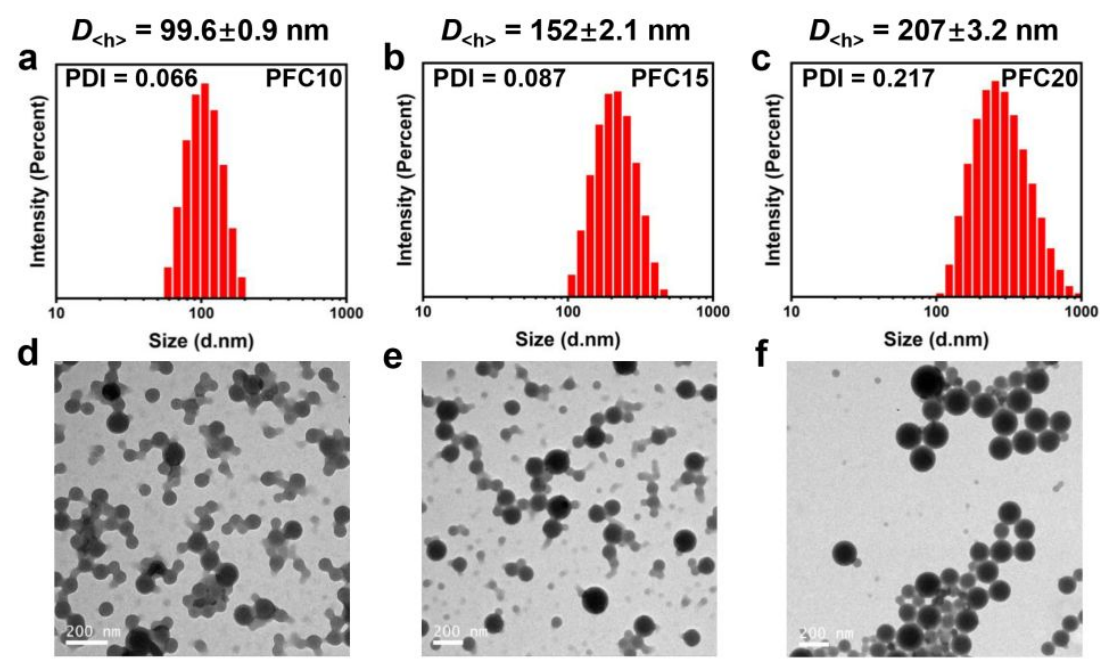

Figure S1 a-c, Hydrodynamic diameter distributions of samples with various diameters; d-f, TEM images of PFC micelles with different diameters. 


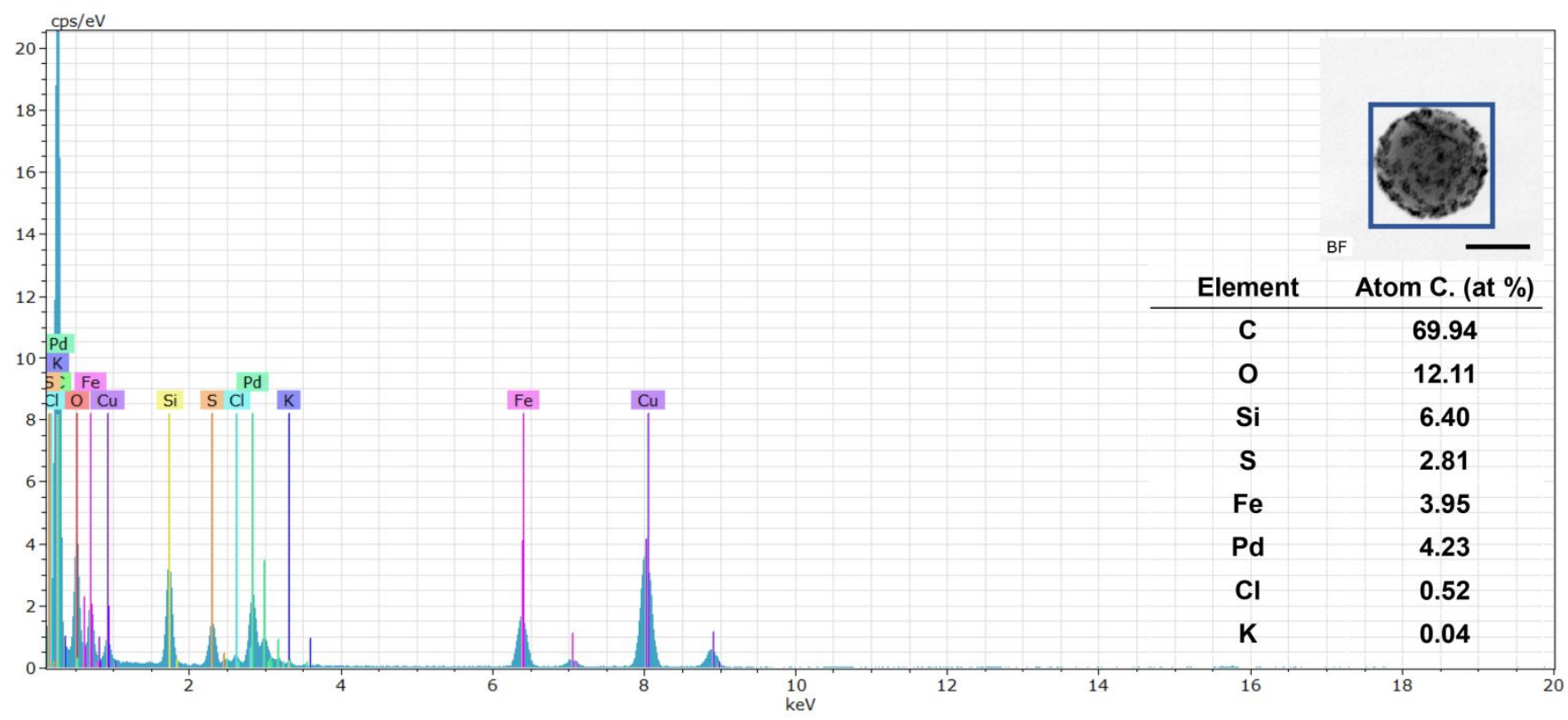

Figure S2 EDS spectrum of PFC/Pd240 NCs. 


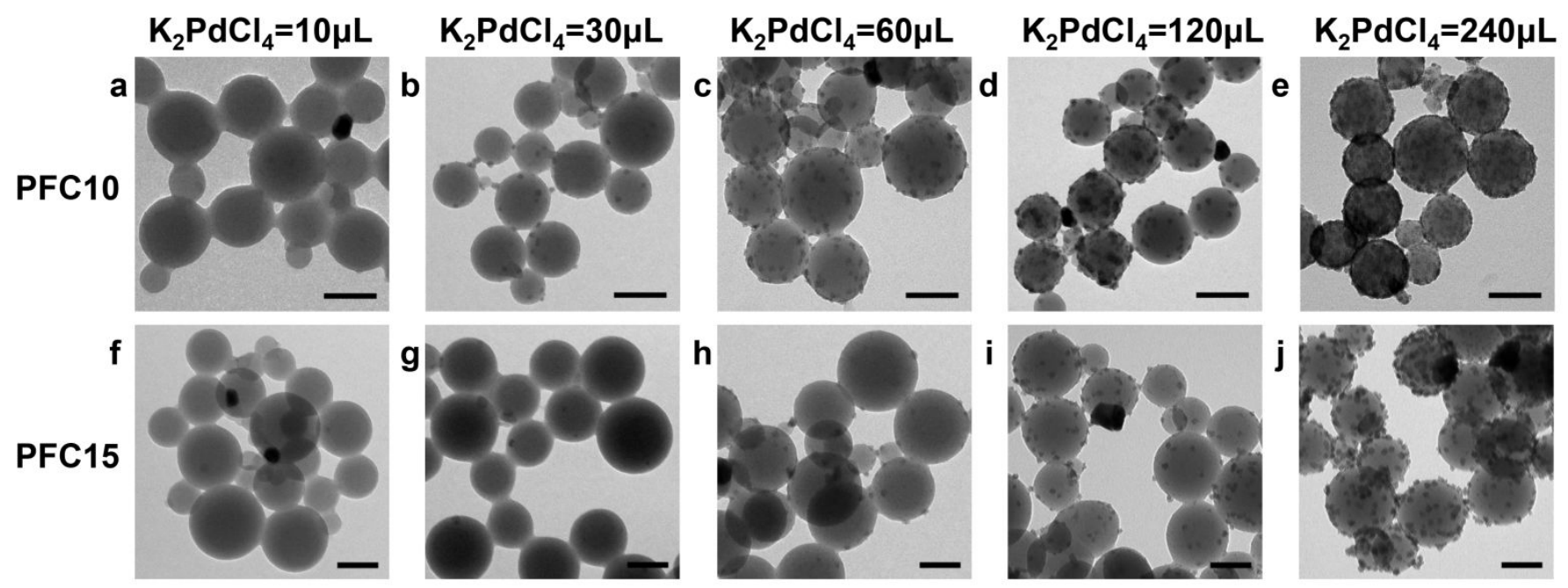

Figure S3 TEM images of PFC/Pd NCs that were prepared with different dosages of $\mathrm{K}_{2} \mathrm{PdCl}_{4}$ using $\mathrm{PFC}$ micelles with a hydrodynamic diameter of $100 \mathrm{~nm}$ (PFC10) as precursors (a-e) and $150 \mathrm{~nm}$ (PFC15) (f-j). Scale bar: $100 \mathrm{~nm}$. 

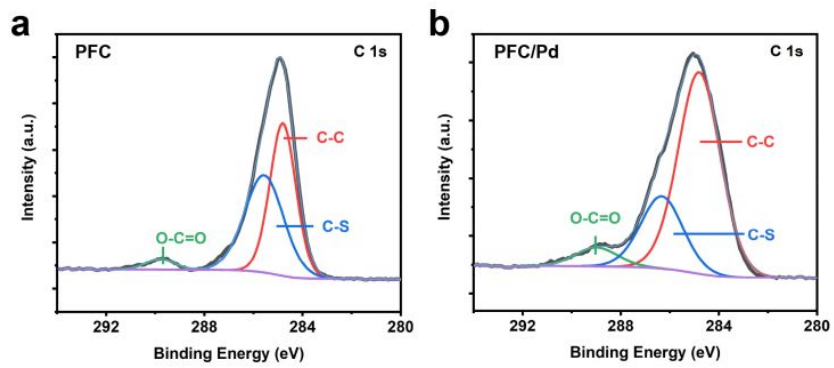

Figure S4 C 1s photoelectron emission spectrum of PFC and PFC/Pd240. 
a

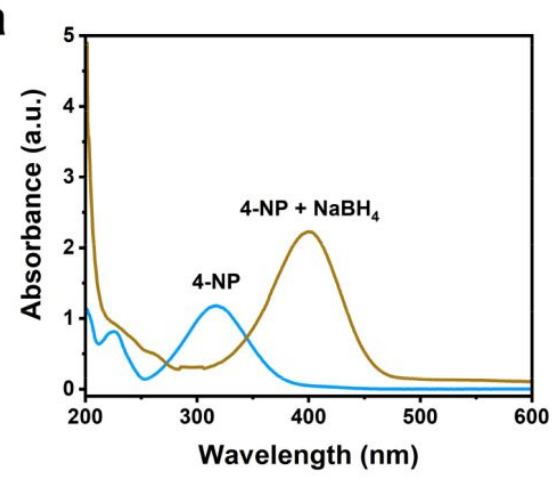

b

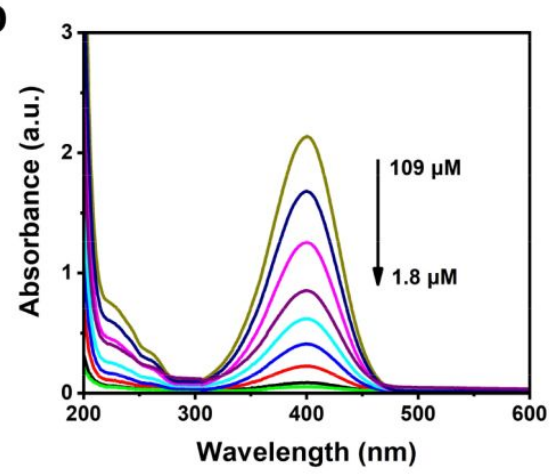

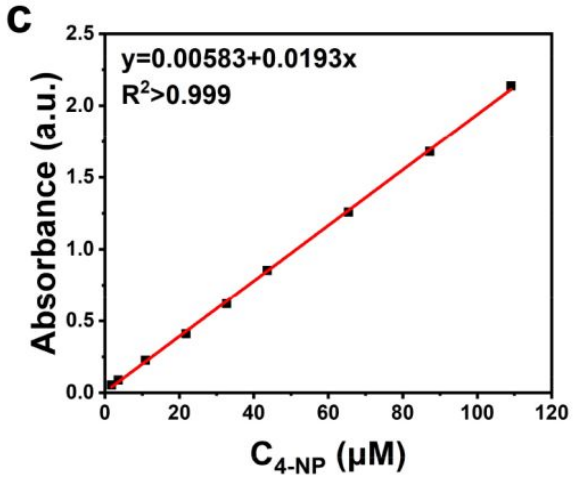

Figure $\mathrm{S5}$ a, UV-Vis spectra of an aqueous 4-NP solution before and after the addition of $\mathrm{NaBH}_{4}$; $\mathbf{b}$, UV-

Vis spectra of aqueous 4-NP solutions at different concentrations in the presence of $\mathrm{NaBH}_{4} \mathbf{c}, \mathrm{Calibration}$ curve of 4-NP with $\mathrm{NaBH}_{4}$ at an absorbance of $400 \mathrm{~nm}$. 


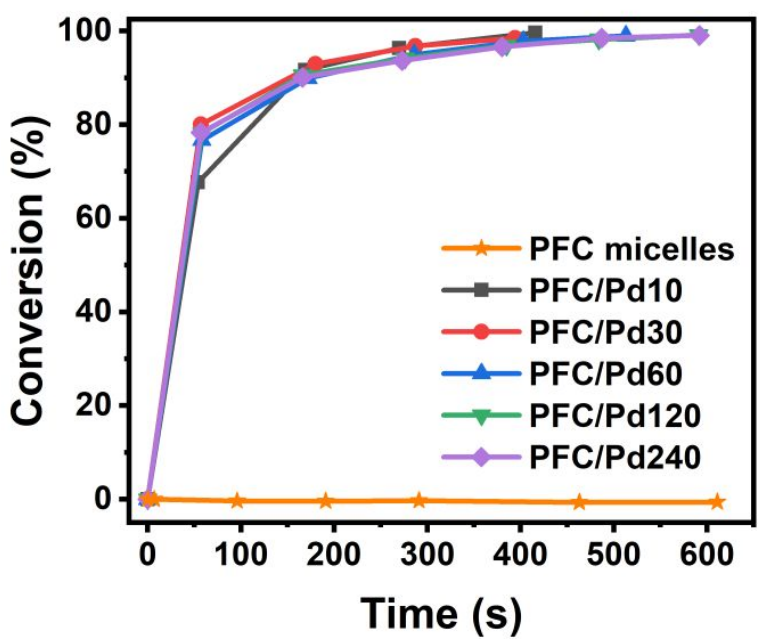

Figure S6 Conversion of 4-NP versus time during the reduction of 4-NP that was catalyzed by the PFC/Pd NCs. 

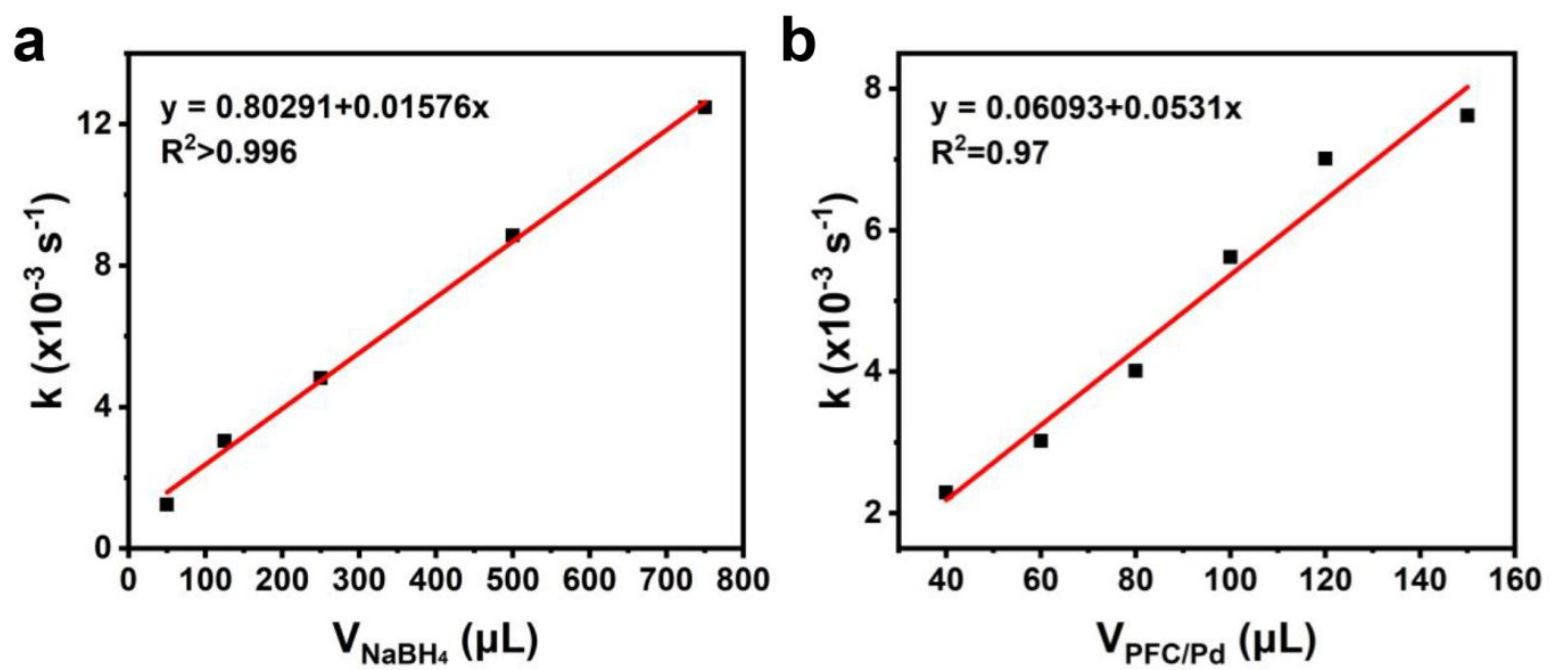

Figure S7 Plots of the apparent rate constant $k$ versus the dosage of $\mathrm{NaBH}_{4}$ and $\mathrm{PFC} / \mathrm{Pd}$. 
Table S1 Catalytic performance of various catalysts based on Pd nanoparticles.

\begin{tabular}{|c|c|c|c|c|c|c|}
\hline Catalyst & $\operatorname{Pd}(w t \%)$ & $\begin{array}{c}m_{\text {catalyst }} / m_{4-\mathrm{NP}} \\
(a)\end{array}$ & $\begin{array}{c}\mathbf{M}_{\mathrm{NABH} 4} / \mathbf{M}_{4-\mathrm{NP}} \\
(b)\end{array}$ & $k\left(10^{-3} \mathrm{~s}^{-1}\right)$ & $\begin{array}{c}k /(a \cdot b) \\
\left(10^{-5} s^{-1}\right)\end{array}$ & Reference \\
\hline $\mathrm{PFC} / \mathrm{Pd} 10$ & 1.4 & 0.96 & 167 & 12.0 & 7.5 & This work \\
\hline $\mathrm{PFC} / \mathrm{Pd} 30$ & 3.3 & 0.96 & 167 & 7.1 & 4.4 & This work \\
\hline $\mathrm{PFC} / \mathrm{Pd} 60$ & 5.5 & 0.96 & 167 & 7.0 & 4.4 & This work \\
\hline $\mathrm{PFC} / \mathrm{Pd} 120$ & 9.1 & 0.96 & 167 & 5.9 & 3.7 & This work \\
\hline $\mathrm{PFC} / \mathrm{Pd} 240$ & 12.4 & 0.96 & 167 & 6.0 & 3.7 & This work \\
\hline $\begin{array}{l}\mathrm{PFC} / \mathrm{Pd} 10 \\
\text { after } 5 \text { cycles }\end{array}$ & 1.4 & 0.96 & 167 & 1.3 & 0.8 & This work \\
\hline $\begin{array}{l}\mathrm{PFC} / \mathrm{Pd} 30 \\
\text { after } 5 \text { cycles }\end{array}$ & 3.3 & 0.96 & 167 & 3.9 & 2.4 & This work \\
\hline $\begin{array}{l}\mathrm{PFC} / \mathrm{Pd} 60 \\
\text { after } 5 \text { cycles }\end{array}$ & 5.5 & 0.96 & 167 & 3.6 & 2.2 & This work \\
\hline $\begin{array}{l}\mathrm{PFC} / \mathrm{Pd} 120 \\
\text { after } 5 \text { cycles }\end{array}$ & 9.1 & 0.96 & 167 & 3.4 & 2.1 & This work \\
\hline $\begin{array}{l}\mathrm{PFC} / \mathrm{Pd} 240 \\
\text { after } 5 \text { cycles }\end{array}$ & 12.4 & 0.96 & 167 & 3.3 & 2.1 & This work \\
\hline $\mathrm{Fe}_{3} \mathrm{O}_{4} @$ Tannic/Pd(0) & 3.75 & 0.58 & 80 & 18 & 38.8 & 1 \\
\hline $\begin{array}{l}\mathrm{Fe}_{3} \mathrm{O}_{4} / \mathrm{SiO}_{2^{-}} \\
\mathrm{NH}_{2} @ \mathrm{CS} / \mathrm{Pd}\end{array}$ & 1.62 & 661.3 & 30 & 12 & 0.06 & 2 \\
\hline Pd@PPM2 & 1.76 & 22.3 & 125 & 8 & 0.3 & 3 \\
\hline $\mathrm{Fe}_{3} \mathrm{O}_{4} / \mathrm{SiO}_{2} @ \mathrm{PDA} / \mathrm{Pd}$ & 1.85 & 4.79 & 100 & 75.2 & 15.7 & 4 \\
\hline
\end{tabular}



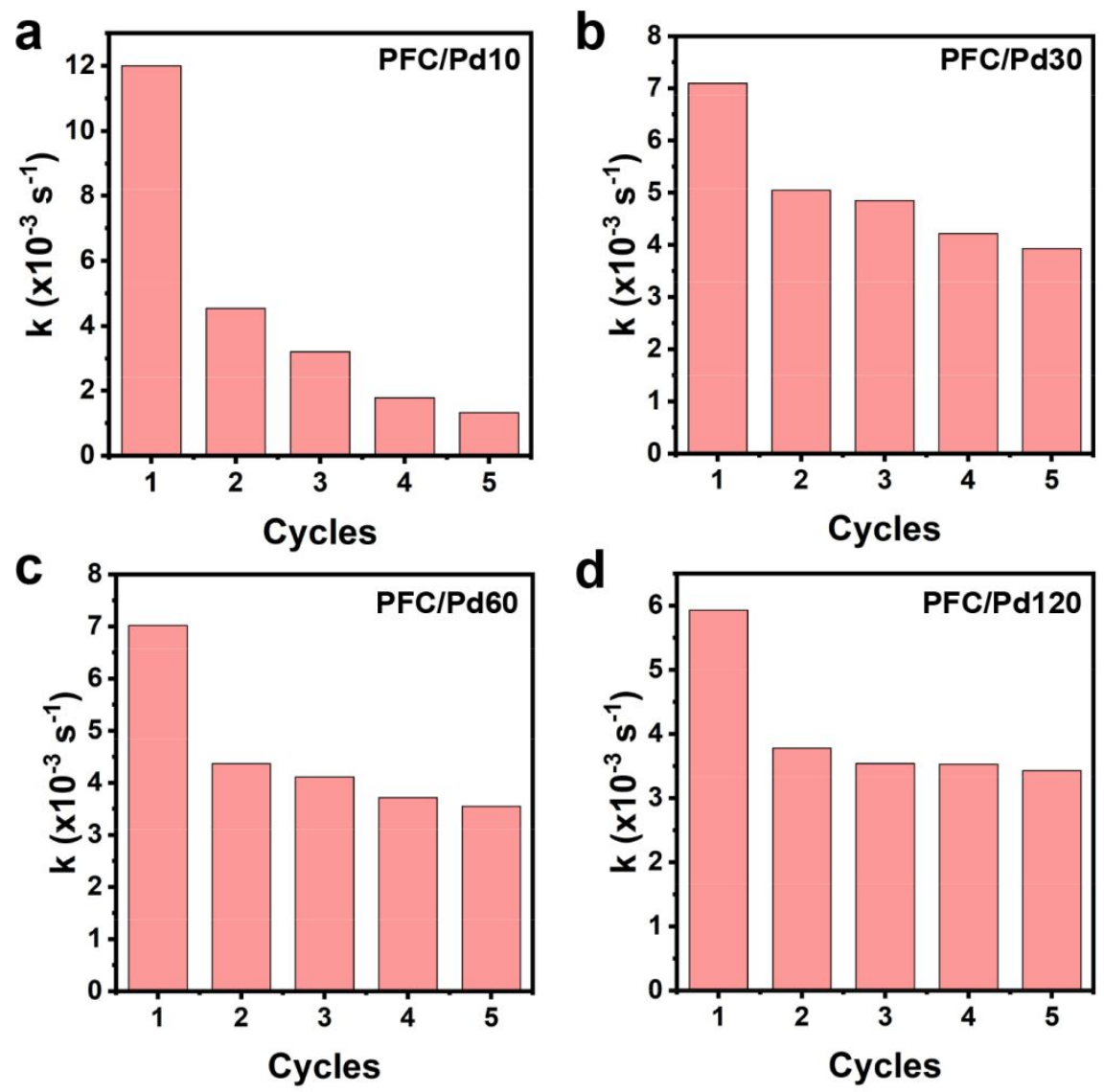

Figure S8 a-d, Variation of the rate constant $k$ during five 4-NP hydrogenation cycles that were catalyzed by the PFC/Pd10, PFC/Pd30, PFC/Pd60, and PFC/Pd120 NCs. 


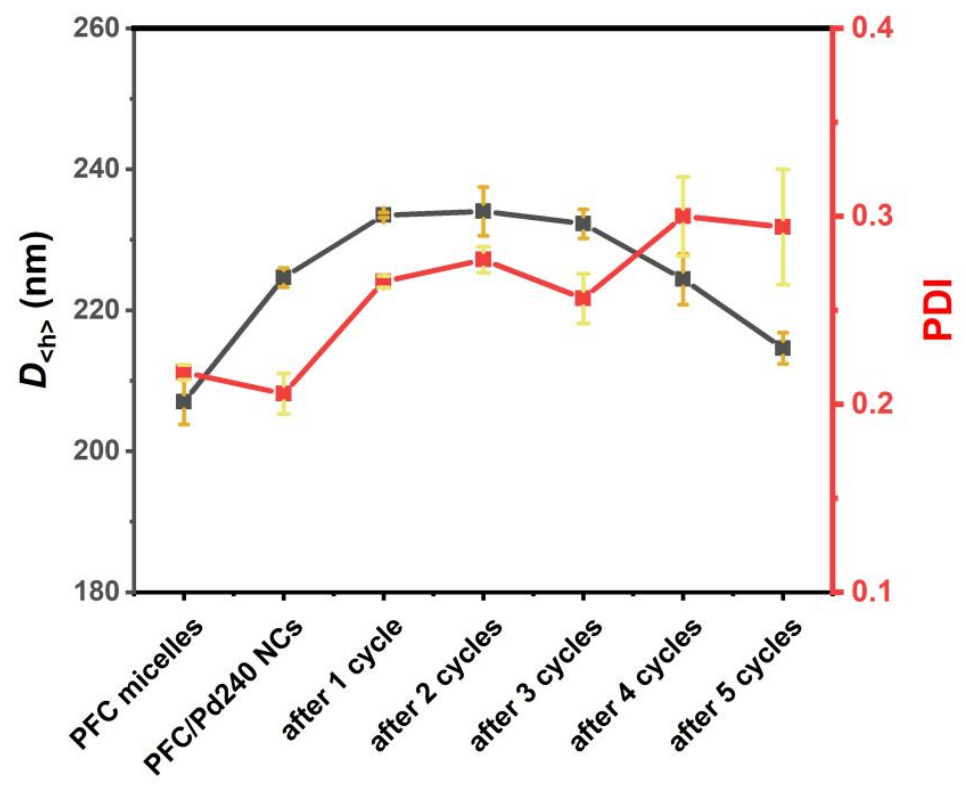

Figure S9 Variation of the hydrodynamic diameter and PDI of PFC/Pd240 during five 4-NP hydrogenation cycles. 


\section{References}

1. Veisi, H.; Pirhayati, M.; Kakanejadifard, A.; Mohammadi, P.; Abdi, M. R.; Gholami, J.; Hemmati, S. In Situ Green Synthesis of Pd Nanoparticles on Tannic Acid-Modified Magnetite Nanoparticles as a Green Reductant and Stabilizer Agent: Its Application as a Recyclable Nanocatalyst (Fe3O4@TA/Pd) for Reduction of 4-Nitrophenol and Suzuki Reactions. ChemistrySelect, 2018, 3, 1820-1826.

2. Veisi, H.; Ozturk, T.; Karmakar, B.; Tamoradi, T.; Hemmati, S. In situ decorated Pd NPs on chitosanencapsulated $\mathrm{Fe} 3 \mathrm{O} 4 / \mathrm{SiO} 2-\mathrm{NH} 2$ as magnetic catalyst in Suzuki-Miyaura coupling and 4-nitrophenol reduction. Carbohydr Polym, 2020, 235, 115966.

3. Bashir, M. S.; Jiang, X.; Kong, X. Z. Porous polyurea microspheres with Pd immobilized on surface and their catalytic activity in 4-nitrophenol reduction and organic dyes degradation. Eur Polym J. 2020, 129, 109652.

4. Farzad, E.; Veisi, H. Fe3O4/SiO2 nanoparticles coated with polydopamine as a novel magnetite reductant and stabilizer sorbent for palladium ions: Synthetic application of $\mathrm{Fe} 3 \mathrm{O} 4 / \mathrm{SiO} 2 @ \mathrm{PDA} / \mathrm{Pd}$ for reduction of 4-nitrophenol and Suzuki reactions. J Indust Eng Chem. 2018, 60, 114-124. 